IJBPAS, November, Special Issue, 2021, 10(11): 1365-1380

ISSN: 2277-4998

International Journal of Biology, Pharmacy and Allied Sciences (IJBPAS)

'A Bridge Betueen Caboratory and QReado'

www.ijbpas.com

\title{
EMBEDDED SYSTEMS FOR AUTOMOTIVE BIO INDUSTRY LABELED COMPOUNDS TO IMPROVE PERFORMANCE
}

\section{VAIBHAW RAMESHRAO DOIFODE ${ }^{1^{*}}$, DANIEL SUNDARRAJ.P ${ }^{2}$, PRASANTH KUMAR.S ${ }^{3}$ \\ AND S.ANUSUYA ${ }^{4}$}

1: Assistant Professor in Electrical Engineering, Yeshwantrao Chavan College of

Engineering, Nagpur, India

2: Assistant Professor \&amp; Head, Department of Computer Science, K.M.G. College of

Arts and Science, Gudiyattam, Tamil Nadu, India (Affiliated to Thiruvalluvar University, Serkkadu, Vellore District - 632 115, Tamil Nadu, India)

3: Research Scholar, K.M G. College of Arts and Science, Department of Computer Science, Gudiyattam, Tamil Nadu, India (Affiliated to Thiruvalluvar University, Serkkadu, Vellore District - 632 115, Tamil Nadu, India)

4: Assistant Professor, Department of Electronics and Communication Engineering, K.Ramakrishnan college of Engineering, Samayapuram, Trichy-621112, Tamilnadu, India

*Corresponding Author: Vaibhaw Rameshrao Doifode; E Mail: vaibhawdoifode1608@gmail.com

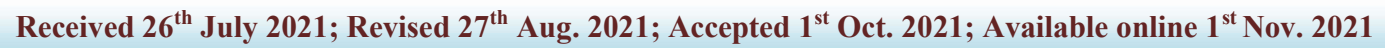
https://doi.org/10.31032/IJBPAS/2021/10.11.1119

\begin{abstract}
A data type, specifically automobile components controlled through channels of remote servers, describes real issues of experiments failures in decentralized integrated circuits which are called ECUs. Authors utilize visualizing research to identify relevant feedback from a variety of validation databases created from tests carried out as part of a systematic operational stage of the software assimilation. Throughout the end, researchers wish to measure the performance of real cause breakdowns and create a consistent communication channel in fault localization. At
\end{abstract}


Volvo Car Organization's (VCC) R and D division, we assess our solution as prepared conditions. The idea helps programming failures succeed by delivering a clear, strong distribution channel and allowing participants can pick and determine the facts on individual displays. Furthermore addition to supports a systematic examination of the regular test standing through the bio industry labeled compounds collection and classification of previous study records from assessment fasteners to reveal linkages in findings.

Keywords: Problem localization; fault information analysis; dispersed integrated circuits;

\section{Bio industry labeled compounds}

\section{INTRODUCTION}

The implementation phase of distributed programs is a significant issue for the automobile sector. Proficient only by observing the interdependencies Competence of other automotive vehicles can be established among both systems inside these automotive vehicles (for example detectors, bundles, and actuators [7] but rather languages of the computer programs and procedures performed upon integrated devices $[5,8]$.

In an automobile system, there are many possible defects. These failures may range from a hardware and software problem with a single subsystem to a communication problem with several subsystems. These errors may be dynamic; for instance, a hardware failure in one subsystem might result in a software failure in another. Two flaws may be indicative of one another [13]. As a consequence of the atypical behavior and many interconnections across different elements, software fault localization may be a very complicated and time-consuming process [6].

Personal experience and subject expertise are required for human fault localization. Because the external expression of the mistake (defect) and its internal source (defect) may have no apparent connection, the job may be extremely costly and timeintensive [1]. Diagnostic methods and scope verification reports often rely on the outcomes of a single test run rather than the information given by many program executions [5, 10]. Furthermore, performance testing results are mainly textual, creating challenges besides larger apps as well as design patterns to comprehend.

Mostly as consequence, designers employ evaluation metrics may functional relationships to discover the deeper reasons for car failures. Moreover, a smarter presentation of compressor stall facts to pick 
and prioritize critical information will assist practitioners to avoid exploring in check trails. Moreover, relation, the representation that has recently been added supports a constant chain reaction mostly in channel estimation workflow, promoting improved model reliability by emphasizing statistics on performance (error/excellence rates) for unit testing. This helps, amongst many thoughts, by implementing guides upon that authoring for unit tests and troubleshooting buffer overrun processes. To improve the efficiency of their fault localization process, our solution provides engineers with a visual interface to examine activation and network behavior. In furthermore, the time requirements offer information on tests in the automotive sector that may be utilized at various phases of the platform and integrations testing process, such as during the design phase.

We want to look at the issues, be much more detailed. The issues are as follows.

RQ 1: If representation seems to be possible, which is used to aid in the identification and localization of faults in automotive system development?

RQ 2: The second question is: How much information collected from automated system development artifacts may be used to help in the process of fault localization?
RQ 3: To most of what degree can visualization provides constant feedback on requirements definition operations the third question.

As a result of the numerous and diverse challenges associated with the prototyping checking of automotive systems, we rely on several assumptions, including These test execution options (screen generating) usually purely objective-based, especially once one building household is associated with deployment tool $I$ as well as the examination will be carried out with competence dynamically (especially family parts on even a circuit).

\section{Literature Survey}

Increasingly important is the existence and importance of software in automobiles, which has become increasingly common. In today's world, an average of 70 networked microprocessors, also referred to as electronic components, are installed in the vehicle's electronics system (ECUs). Throughout relating the ECUs, the sensor readings, as well as binary communications, should have been sent by 5 or more different cellular connections \& received in actual time [6]. Increase exact position is found to be involved modules only with the provision of high-quality features in modern automobiles also including control system 
with increased braking [4] systems has emerged as a topic of interest for researchers in both industry and academia.

Preliminary research on effective energy conversion assessment one by [10], and they offer electrical energy and power infrastructure inside a vehicle [6], who devised a multilayer system to analyze hardware, software, and their relationships. In contrast, our study was tested in a car level components testing and operating system, while their method was verified via the use of a vehicle model;

Introducing a verification method for testing connected vehicle signal processing functionalities, The researcher [2] represent a potential approach focusing on finding test gaps and inconsistencies in a distributed vehicle embedded computing environment. They would not, nevertheless, address the discovery or forecasting of underlying causes of testing failures, which are important considerations.

In particular, offer a way of monitoring audit logs and collecting crucial data to find that fault location [9]. Their work on parsing, categorizing and interpreting existing data to automate the discovery of failure reasons is extremely important to our work, but that is not done in the more complicated context of widespread integrated circuits in the automobile sector, as does ours.

The investigation result suggests an interconnected with reliable source is diagnostic evaluation composed of 6 paths: concept, meaning, development and current evaluation techniques, inductive learnercentered approach, and forecasting [12]. A study on computerized configuration management using Jenkins. In a similar way the author [6], investigators don't test their hypotheses in a real-world manufacturing setting [14-17].

After taking into account the current literature on visualization used in signal processing construction, The investigator [5] present a novel method that makes use of visualization to aid in the localization of faults in embedded systems. Each program statement's involvement in the result of program execution is graphically represented by the use of color, according to the authors of the paper. Although their approach is helpful in terms of fault localization, the scope of their solution is considerably smaller since it focuses only on lines of code that have been executed. As a result, their method would be restricted in its use during test execution, when errors might arise mostly from software problems, and mostly from the proposed method, technology, and 
connectivity links amongst or among its multiple parts.

The issue of finding problems of a breakdown has been handled by the vast majority of the researchers mentioned in the preceding part of this paper. For example, the authors $[2,6]$ explore various methods and models to tackle the problem, with the majority of the discussion focused on simulation. The particular about decentralized integrated systems, researchers are aiming to create and establish patterns from discrepancies within them via the approach that takes trial files including unsuccessful incident logging [9]. Due to the massive reasonable speed of VCCs data collection, authors seem unable to duplicate [7] approach.

Consequently, we focused on the identification of reliability analysis into aspects and learning experience via a strategy to find patterns to divide relevant findings. Data visualization is a developing area in software engineering across a variety of domains, particularly automotive and embedded systems although research into data visualization for testing in those specific domains is still in its early phases, As evidence revealed, the article [12] provides helpful information on fundamental advanced analytics concepts and methodologies, which does not include intricacies of said activities of systems engineering facts, which seem to be a drawback.

\section{METHODOLOGY}

For this current research, we will use a design science approach [4], in which a relevant business issue will be explored via the iterative building of an artifact, allowing us to respond to our research objective. The study is carried out on-site at the Volvo Car Company (VCC), a major automobile company, in the $\mathrm{R}$ and $\mathrm{D}$ section. A team at VCC tasked with optimizing the standardized testing process for networked integrated circuits, particularly automotive systems managed by Electronic Control Unit networking (ECU's). That notion of such connection of learning organizations is used as earlier as feasible for configuration management $(\mathrm{CM})$. Unique elements of the CM pipeline might have autonomous/continuous evaluation that eliminates overhaul-related constructionrelated problems [3-4]. The study is being utilized as the encrypted channel, and inhouse built systems engineering framework is being used in conjunction with it [1]. Figure 1 depicts a high-level overview of the present fault localization procedure. Overall, Jenkins coordinates as well as carries out all 
the deployment of testing techniques involving a range of acceptable reliability with modern technological deliverables. This same configuration management technology produces a unique insight of the positives and negatives of such training dataset, meanwhile, the approaches to managing present the test data findings. To discover and begin manual debugging procedures, technical experts utilize the test results to find failed test cases.

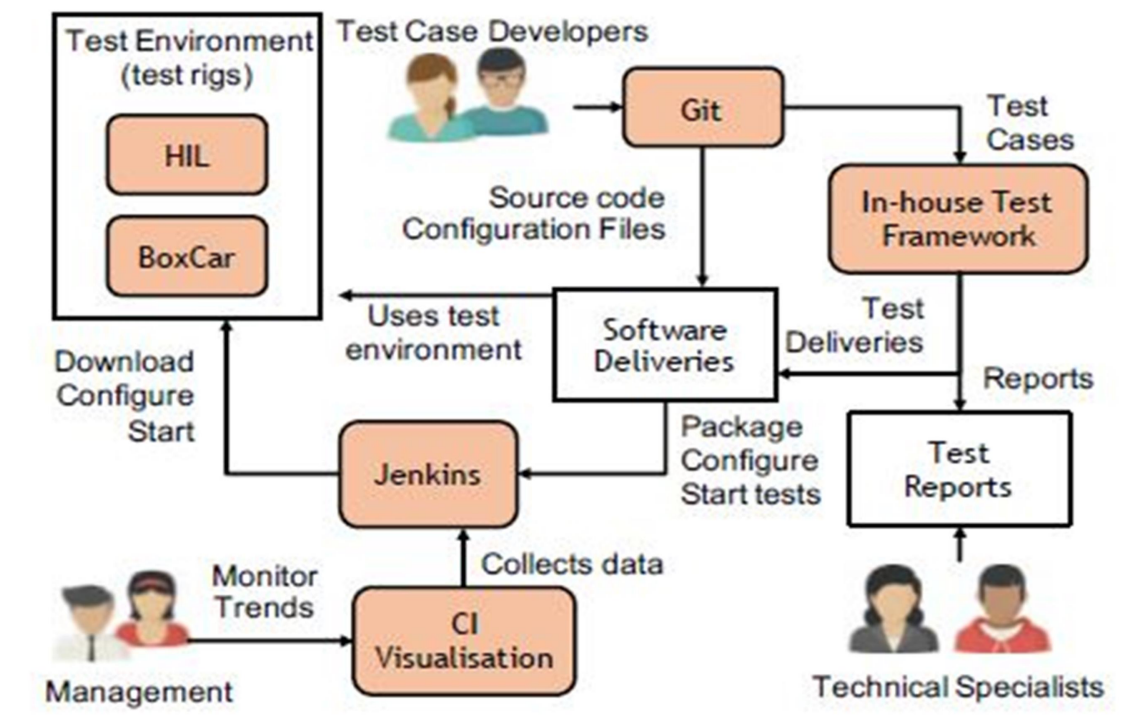

Figure 1: Systems and participants optimized in the CI Methodology

To carry out the process outlined above, in-house specialists must be able to assess the root of the issue of fails depending mostly on evolving broadcaster's previous experience and skill, as well as the results of test reports, contains a vast amount of information about all system testing those which did not attempt.

In intended to facilitate experts by having provided sensible malfunction relevant data, researchers conducted interviews with the relevant to the job for the manual categorization of failures to capture components of their tacit and explicit knowledge of ability to handle the failure Basic content has been used to construct decision-making bodies utilizing CMPipeline certifications to create some sort of such hand-operated categorization \& (1) the appropriate findings from its assessment. Thus, research progress includes: I am a precedent-setting decomposition classification tree; (2) I am systematically explaining trial logs; (3) I retrieve as well as replace a given dataset instantly using 
development tools; and (4) an application development concept using data gleaned.

That program was constructed as well as constructed inside of an evolutionary sense that according to proposed research [4] and was technique then regularly shown to consumers while de-sicking. The resultant platform was subjected to a thorough evaluation and was represented from both a technological and a managerial standpoint.
Integrating engineers, project managers, and other stakeholders were able to understand and interpret the test findings via the use of appropriate graphs and plots created specifically for this purpose. Each of the four components of the data presentation tool is responsible for a specific task: (1) test information collection, (2) test data storage, (3) accessing of collected data, and (4) visualization.

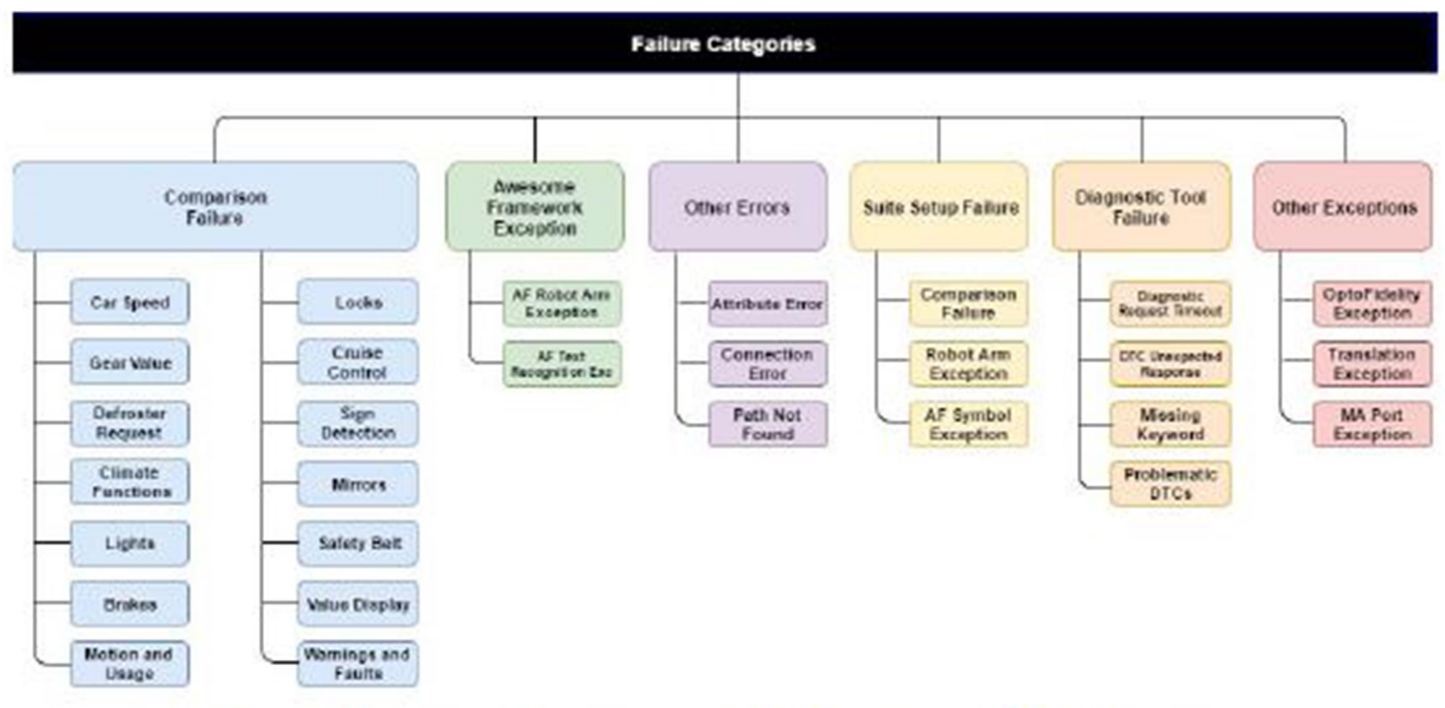

Figure 2: Malfunction patterns categorization

Data Acquisition and Processing for 250 applications, representing a considerable Testing

These authors made adjustments toward a substantial number of known recordings executed throughout a configuration management procedure using a massive operating system along with functional testing. Approximately 35 design patterns comprised a maximum with over quantity of evidence, comprising 11, 500 system testing reports (six months during 2016 and 2017). Furthermore, developers had capable of triggering \& collect current data through tests wherever and whenever necessary through connectivity to that same CI architecture. In particular, the unit test description, prompt traces, or the database of 
configuration files triggers each test $[\mathbf{1 1}, \mathbf{1 3}]$.

Those who were made test cases have succeeded or missed. We also conducted interviews with team members who were experts in the potential underlying causes of particular test failures and who shared their expertise with us.

Verification and validation reports comprise the major form of information gathering, even though they are created also by Automation technique 2 procedures used by investigators of all this investigation.

Such records are typically created also with application HTML and Powerful Query Languages (XML), mostly with recommended version generally HTML.

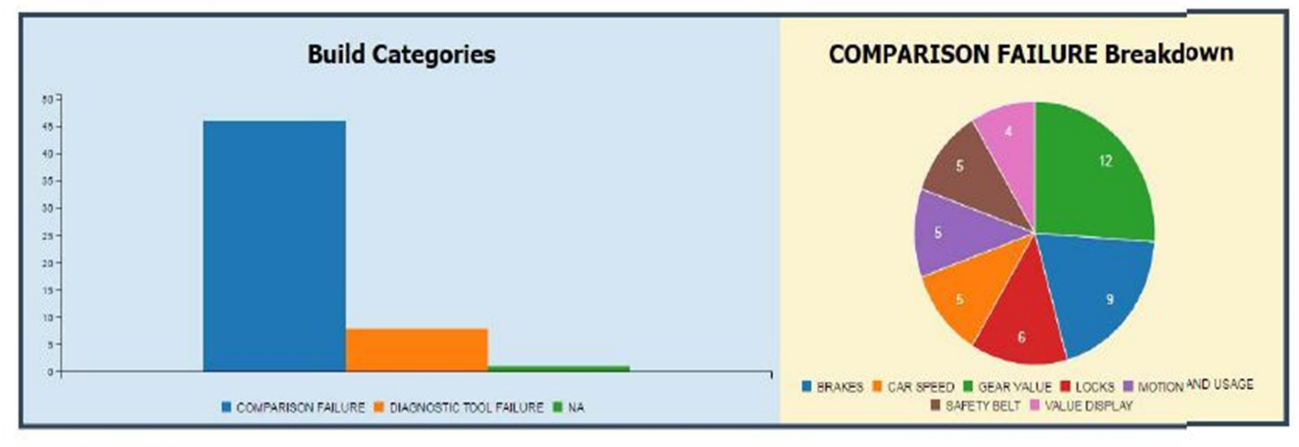

Test Case Failures

\begin{tabular}{|c|c|c|c|c|c|}
\hline Test Suile & $\begin{array}{l}\text { Tet } \\
\text { Case ID }\end{array}$ & Test Case Naine & Categary & Warn Message & Fall Message \\
\hline HIL * HIL stabity $94032>$ Bascoriving Cyde & $51 \cdot 52 \cdot .5$ & $\begin{array}{l}\text { Simple divive cyde - } \\
\text { slow acceleration - } \\
\text { soft braking }\end{array}$ & $\begin{array}{l}\text { COMPARISON } \\
\text { FALURE }\end{array}$ & $\begin{array}{l}\text { FAlLED CHECK: car speed is areater } \\
\text { than or equal to [10]] }[10.0- \\
\text { Adtual value: } 2.53368[\mathrm{~m} / \mathrm{s}]\end{array}$ & Compartson falure \\
\hline $\begin{array}{l}\text { PKE > PKE Ve fied > PKE } 017 \text { S-17-04 } \\
\text { GearPostionWaming }\end{array}$ & $51-64 \cdot+1$ & $\begin{array}{l}\text { S-17-04 Gear } \\
\text { Poston Warming }\end{array}$ & $\begin{array}{l}\text { COMPARISON } \\
\text { FAIUURE }\end{array}$ & 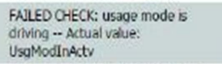 & Comparison falure \\
\hline $\begin{array}{l}\text { PKE > PKE Verfied }>\text { PKE } 017 \text { s-17-04 } \\
\text { GearPOSstionWaming }\end{array}$ & $51-54 \cdot 12$ & $\begin{array}{l}\text { Dsengage EPB } \\
\text { and drive of }\end{array}$ & $\begin{array}{l}\text { COMPARISON } \\
\text { FALURE }\end{array}$ & $\begin{array}{l}\text { FAlED CHECK: electncal parking } \\
\text { brake status s released - Actual } \\
\text { value: applied }\end{array}$ & Comparison Aaure \\
\hline
\end{tabular}

Figure 3: Data visualization displays an overview for failures

Regardless of its readability, the after a comprehensive study among all HTML style is more suitable for human usage, while XML is more suited for computer reading. This resulted in the development of XML-friendly technologies that fetched, collected, and analyzed the data anytime.

\section{Categorization of Failures plus facts}

\section{Interpretation of Results}

Each design including its failed failings including its prototype model \& interrogation events.

Initially, the malfunction type was employed as the primary categorization criterion, and it was subsequently segmented based on other factors, such as the organizational unit impacted or the ECU to which it was connected. Several important considerations were factored into the categorized structure was only established 
equation while creating the visualization template:

- The first emphasis will be installing the initial findings.

- Any proper planning description featuring, whether relevant, current verification code. • A concise summary of its medical reports shown possible causes about a mistake, as diagrams.
Informative listing of failing pilot projects but it is much easier to comprehend than those of the academic performance logging public Knowledge of new problems.

- Such a spreadsheet gives data on frequency errors that allow the number of parts and indeed the $\%$ of unsuccessful cycles to sort.

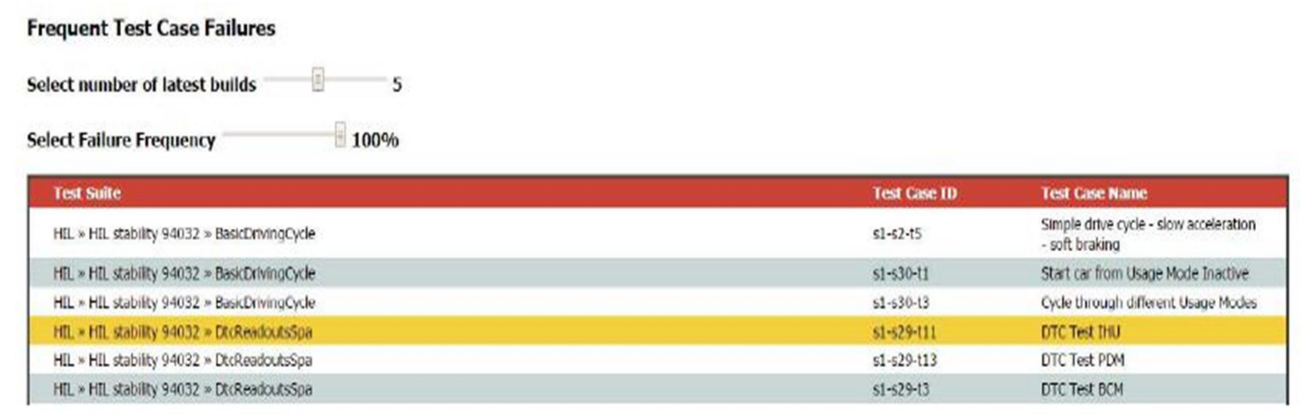

Figure 4: Visualization historiography failures site, newer and recurrent failures

\section{Appraisal of the situation}

That was their objective must carry out a visual inspection including its specific campaign as analyzing what the artwork created relates also with existing representation; throughout accomplishing such, suggested strategies picked experienced familiar again with previous VCC setting. The latter enables us to explore and compare perceptions of even the systematic technique that would have been extremely useful for the responders. Consequently, despite the small number of participants available, descriptive statistics were not able to be used in our research.
As a result of doing moderate conversations with open-ended and return the questionnaire depending on the subjects that require attention, we were able to dig further into the answers than we could have otherwise. Although a list of questions was prepared in advance of the interviews, the researchers were given the flexibility to ask the interviewer spontaneous questioning based on their answers during the process to foster a sense of community between the respondents and the investigators.

Several respondents were chosen to give input from a variety of stakeholder groups, including the test rig accounting 
department ( 2 persons, 1 joint meeting), the continuous deployment team leader, and the configuration management team (1 person, 1 joint meeting). Numerous stakeholders offered perspectives on both the management and engineering aspects of the project. The team member gave an overview of how well the inspection process was doing, while the experimental setup analyzer was more preoccupied with the elements that were specific assemble as well as locate an actual problem in each machine yourself. Authors have significant statements as well as done a themed analysis helping to improve every respondent's understanding of remarks upon this novel visualization technology. After the research, the findings of the dates were presented and debated with the parties involved.

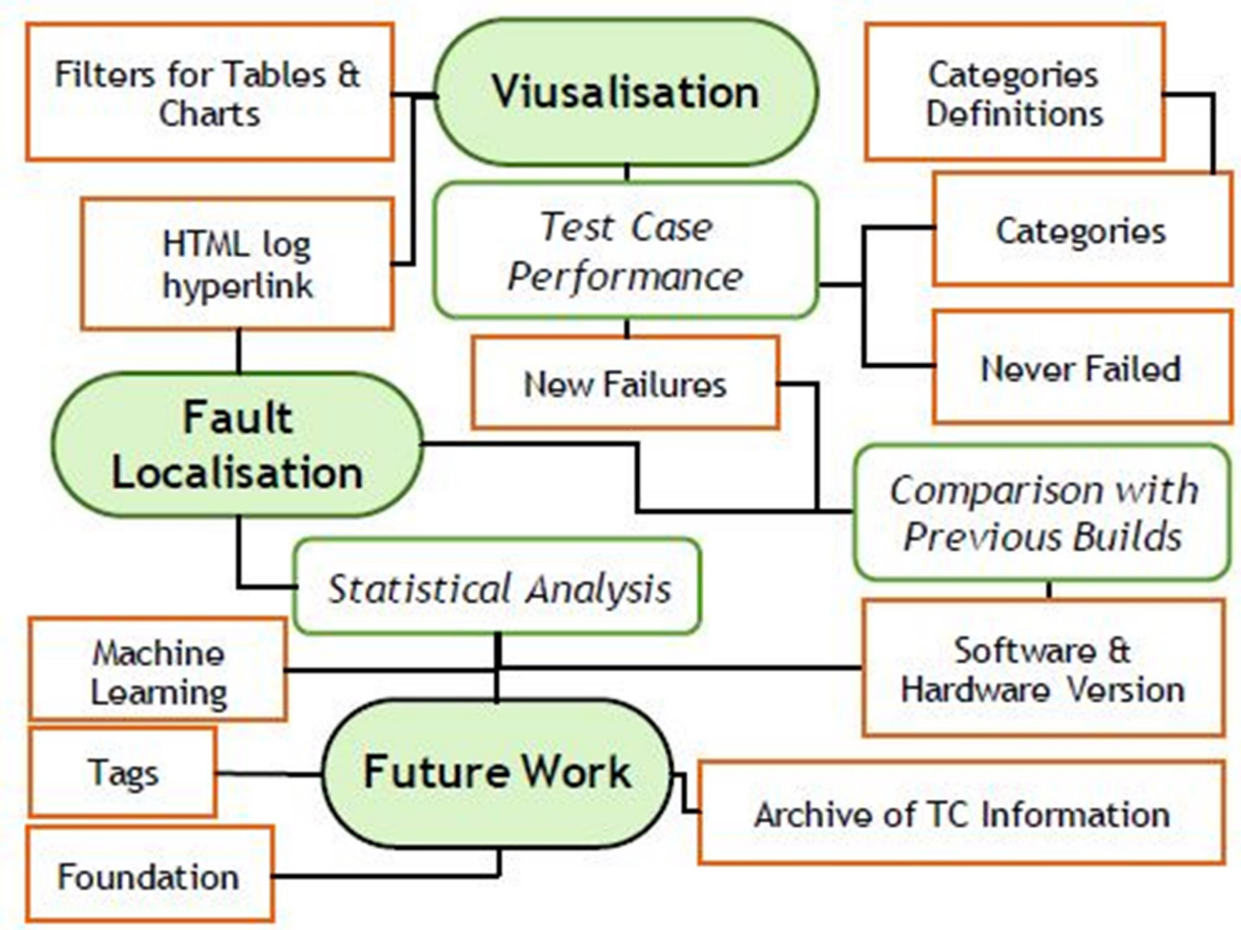

\section{OUTCOMES}

\section{Tree of Postoperative complications}

The divisions inside this error classification model were generally concentrated mostly on subjective categorization performed physically by the professionals who were questioned for this project. The categorization is linked to components from its testing logs, allowing testers to dig further into the scripting to identify specific issue regions that occurred during the execution. The important knowledge required to categorize different failure kinds is thus captured in each 
category, as is the data that can also be retrieved from either the testing summary. Throughout particularly, the warnings and failure communications generated by the new tests were thoroughly examined, and divisions and sub-categories were created by the degree to which the messages differed from one another.

Using this tree, the content from sample analysis may be immediately deemed and segregated, allowing human specialists to carefully examine different kinds of errors or the details about them, for example, implementation patterns, estimated and practical outcomes, and its performance measures). Three degrees of failure were used to categorize failures:

Phase 1 - Failure Group: The whole step classifies several components depending upon on significance and role of malfunction. Other defects include comparability breakdown (unless a claims assessment misses) \& troubleshooting utility breakdown (if a troubleshooting program has an error).

Phase 2 - Failing Breakdown: This level characterizes the many kinds of failure by identifying common probabilities among them. Such comparability flaws get categorized by another structure portion (driver behavior, braking, clamps, etc.), whereas Troubleshooting Device faults are typically segmented by ECU which just caused that error details and some other poor contact with other components.

Fault Visualization technique (Visualization of Failure)

Each photograph in the visualization technique has been used in this form of internet sites that were produced by Jenkins on the fly. The script was written by the investigators and included in the test executions that they conducted. It is possible to interact with the data on the pages (Figures 3 and 4) to quickly identify the most important information required to discover the probable root cause(or) of detailed test problems.

The failures representation is generated using a mix of performance testing results, the failure logic model, and standard deviations (mean, standard deviation, and so on) from prior builds, all of which are combined. The bar chart allows the viewer to communicate with both the data by clicking on a column heading, which updates the frequency distribution and tables of test case rejections with the most recent relevant information.

Secondly, this same program helps describe exactly the way your research model works across an extended time frame, which is displayed in Figure 4. and also it will 
enable specialists to go back in time and detect patterns in test executions over time. As a result, they are provided with the following: I A table containing data sets who had never failed, and (ii) an integrated validation column which has now frequently attempted all across the more likely number consecutive iterations. It further includes an unsuccessful wavelength reduction that decreases the potential for error like 20 percent, 40 percent, 100 percent, happening. Analysts can choose, besides instance, that examine a selection of feasibility studies that have fallen fifty percent compared to the previous 3 constructions. Taking a long-term view of lab results information in the context of networked integrated circuits should emphasize to both programmers and other customers which aspects of the infrastructure needs need to be upgraded to enable more reliable strong reliable in the future.

\section{Analysis}

Throughout this section, researchers address our research objective RQ1 through RQ3 after coding and categorizing the research findings into thematic groups. Figure 5 depicts the connection between the retrieved codes and the associated themes/sub-themes that they represent. The following are the themes and sub-themes that have been identified:
- Error detection (RQ1 and RQ3) - Captures all elements of the process of finding problems inside the computerized evaluation process, includes various electronic control information systems altogether agree on both). "Representation" relates throughout this sense toward the dedicated service generated and all the information regarding that included in the domain and also agree on RQ2.

- Study intends to describe tracking solution constraints and highlight prospective enhancement towards the usefulness of said prototype that agrees with all.

The term "detailed test effectiveness" refers to the sum of all elements of how test cases perform during a period of one or more deployments that agreed on RQ3.

Using the current build to compare it to previously performed builds to demonstrate commonalities or differences is known as comparison Previously built is agree on RQ1 and RQ2. Everything just provides statistical technology to monitor a building design to predict or communicate ideas that RQ3.

The much more important characteristics within their proposal, including customizable tables of diagram controls and enable participants to pick just the necessary information they are interested in, were also recognized by participants. 
When compared to the prior method employed at VCC, that provides a great deal of energy for consumers by removing extraneous check errors but also to show just situations that need to be bothered with. However, the participants were worried that the visualization would eliminate an excessive amount of information.

Interestingly, the management viewpoint from the project manager participant expressed an Attraction throughout examinations which have never flopped, but specialists mostly on prototype showed high involvement in original error findings. This thing is possible to purchase the most recent build with prior builds was the most often mentioned topic in both interviews, and it remains so today.

When asked about statistical techniques of test data, the CM team manager replied that they play a significant part in the process of fault localization. According to the results of this interview, our efforts laid a strong basis for VCC's ongoing move toward completely automated assessment and fault localization.

\section{Based on the findings of the interview:}

RQ1: The ability to categorize and show failures as charts are provided. Test rig experts may more efficiently detect problems that need urgent attention by using advanced technology. Being the ability to identify and filter out failures by some of these regions assists them in concentrating on what has to be done treated first and foremost.

RQ2: Optimization of the quantity of data collected for each test case Introducing connections for fine-grained data decreases the amount of time it takes to read. Different positions in the organization see an abundance of redundant data in the course of action.

RQ-3:

Process planning contains data from many sources that appear on one webpage, minimizes the amount of effort of such authorities since they should not have to browse across several databases that gain knowledge it is complete concerning method based.

\section{Threats to the Validity of the Hypothesis}

One of the most serious challenges to our study's internal validity is the limited fraction of participants and interviews were conducted throughout the study. The information is being evaluated. It was, on the other hand, safer from the company's viewpoint, it makes sense to conduct this first research in modest sample size and scale. This danger was minimized by appointing senior members to the committee, that may be shared by the team (test analysts, experts, 
and the CI leader). A significant amount of information regarding the trade-offs associated with our prototype. When it comes to external legitimacy, the most dangerous is that the research was carried out at a single automobile manufacturer. So findings may not be repeatable in other businesses and may not be applicable. Because of the NonDisclosure Agreement, the study's findings can indeed be made accessible. But on the other hand, the vast majority of our findings are based on the claimed advantages of utilizing this product. Graphical representations and data-driven methods are used to ensure that our findings are actionable. That may be compared to previously published research in the literature. Finally, we used a methodical approach while creating the fault tree. Categories are constant inside their subdivisions, and they are consistent overall. However, it is not sufficiently comprehensive over the same level. Regardless, this effort is a first step toward the establishment of a CI culture. From either the company's viewpoint, it is safer to keep the information confidential. Start with modest, lower-risk research with a smaller number of respondents as well as a restricted reach inside the organization itself.

\section{CONCLUSION AND OUTLINE FOR FUTURE WORK}

Our research team has developed a unit test failures performance analysis system that may be used to assist with problem localization processes in the automobile industry. Such application development review is performed simply merging an unsuccessful decision tree alongside histories filter was designed, which comprises the outcome including its latest tests performed also historical information about partially executed trials. The failures are classified and represented to allow for fast presentation and accessibility of the test findings once they have been completed.

In research, we assess our suggested solution by interviewing the key stakeholders to determine the impact of the visualization method on their perceptions. As several functionalities have been stretched or assigned to interpret well there significantquality sensor readings including the differentiation of rendition by technologies of such plant growth parameters, also every finding already has shown to be the recently approved computer configuration does have a positive effect, mainly upon on faulty equipment translated text.

Future work will involve the expansion of the model to incorporate those 
new elements, as well as the expansion of the study results scope to encompass other VCC initiatives to consolidate existing findings.

\section{REFERENCES}

[1] L. C. Ascari, L. Y. Araki, A. R. T. Pozo, and S. R. Vergilio. Exploring machine learning techniques for fault localization. In 2009 10th Latin American Test Workshop, pages 1-6, March 2009.

[2] A. Chunduri, R. Feldt, and M. Adenmark. An effective verification strategy for testing distributed automotive embedded software functions: A case study. In ProductFocused Software Process Improvement, pages 233-248. Springer International Publishing, 2016.

[3] F. G. de Oliveira Neto, A. Ahmad, O. Leifler, K. Sandahl, and E. Enoiu. Improving continuous integration with similarity-based test case selection. In Proceedings of the 13th International Workshop on Automation of Software Test, AST '18, pages 39-45, New York, NY, USA, 2018. ACM. ISBN 978-14503-5743-2.

[4] A. R. Hevner, S. T. March, J. Park, and S. Ram. Design science in information systems research. MIS Q., 28(1): 75-105, Mar. 2004. ISSN 0276-7783.

[5] Devaraj S, Malkapuram R, Singaravel B. Performance analysis of micro textured cutting insert design parameters on machining of Al-MMC in turning process. International Journal of Lightweight Materials and Manufacture. 2021 Jun 1;4(2):210-7

[6] Arunkarthikeyan, K., Balamurugan, K., \& Rao, P. M. V. (2020). Studies on cryogenically treated WC-Co insert at different soaking conditions. Materials and Manufacturing Processes, 35(5), 545-555.

https://doi.org/10.1080/10426914.2020.1 726945

[7] Arunkarthikeyan, K., \& Balamurugan, K. (2020). Studies on the effects of deep cryogenic treated WC-Co insert on turning of A16063 using multi-objective optimization. SN Applied Sciences, 2(12), $1-14$. https://doi.org/10.1007/s42452-02003940-3

[8] Garigipati RK, Malkapuram R. Characterization of novel composites from polybenzoxazine and granite powder. SN Applied Sciences. 2020 Sep;2(9):1-9.

[9] Yarlagaddaa J, Malkapuram R, Balamurugan K. Machining Studies on Various Ply Orientations of Glass Fiber Composite. InAdvances in Industrial Automation and Smart Manufacturing 2021 (pp. 753-769). Springer, Singapore.

[10] Sridharan, K., \& Sivakumar, P. (2018). A systematic review on techniques of feature selection and classification for 
text mining. International Journal of Business Information Systems, 28(4), 504-518.

[11] Vemuri, R. K., Reddy, P. C. S., Kumar, B. P., Ravi, J., Sharma, S., \& Ponnusamy, S. (2021). Deep learning based remote sensing technique for environmental parameter retrieval and data fusion from physical models. Arabian Journal of Geosciences, 14(13), 1-10.

[12] Venkata Pavan, M., Karnan, B., \& Latchoumi, T. P. (2021). PLA-Cu reinforced composite filament: Preparation and flexural property printed at different machining conditions. Advanced Composite Materials, https://doi. org/10.1080/09243046.2021, 1918608.

[13] Garikapati, P., Balamurugan, K., Latchoumi, T. P., \& Malkapuram, R. (2021). A Cluster-Profile Comparative Study on Machining AlSi $7 / 63 \%$ of SiC Hybrid Composite Using Agglomerative Hierarchical Clustering and K-Means. Silicon, 13, 961-972.

[14] Yarlagaddaa J, Malkapuram R. Influence of carbon nanotubes/graphene nanoparticles on the mechanical and morphological properties of glass woven fabric epoxy composites. INCAS Bulletin. 2020 Oct 1;12(4):20918.
[15] Rama Krishna M, Tej Kumar KR, DurgaSukumar G. Antireflection nanocomposite coating on PV panel to improve power at maximum power point. Energy Sources, Part A: Recovery, Utilization, and Environmental Effects. 2018 Oct 18;40(20):2407-14.

[16] Arunkarthikeyan, K., \& Balamurugan, K. (2021). Studies on the impact of soaking time on a cryogenic processed and post tempered WC-Co insert. Materials Today:

Proceedings, 44, 1692-1699. https://doi.org/10.1016/j.matpr.2020.11. 869

[17] Arunkarthikeyan, K., \& Balamurugan, K. (2021). Graph Theory and Matrix Approach for Machinability Enhancement of Cryogenic Treated Cobalt bonded Tungsten Carbide Inserts, International Journal of Heat and Technology. 